\title{
The Sensitivity of Cells from Normal Mouse Bone Marrow to Gamma Radiation in Vitro and in Vivo'
}

\author{
E. A. McCULLOCH AND J. E. TILL \\ Department of Medical Biophysics, University of Toronto, and the Divisions of Biological \\ Research and Physics, The Ontario Cancer Institute, \\ Toronto, Canada
}

\section{INTRODUCTION}

Normal mouse bone marrow contains a small population of cells with the capacity to give rise to macroscopic colonies containing differentiated hemic cells in the spleens of heavily irradiated animals. A radiation survival curve for these colonyforming cells after irradiation in vitro has been published (1). However, it remained to be shown whether this survival curve is applicable to colony-forming cells irradiated under physiological conditions in the animal. In the present paper, a survival curve for the colony-forming cells irradiated in vivo is presented and compared with the in vitro survival curve. Although these two curves are very similar, a significant difference does exist between them.

\section{EXPERIMENTAL PROCEDURES}

\section{Measurement of the Colony-Forming Ability of Marrow Cells}

The methods used to test for the colony-forming ability of cells in a marrow suspension have already been described (1). In brief, known numbers of nucleated cells from the femoral marrow of mice are injected intravenously into heavily irradiated (900 to 1000 rads) recipient animals. After 10 days, surviving animals are sacrificed, and their spleens are fixed in Bouin's solution (2). Fixation in Bouin's solution causes any macroscopic colonies that are present to stand out against the darker background of the host's splenic tissue as round or oval yellow nodules, which may readily be counted. Satisfactory counts can be made with as many as 25 colonies per spleen. However, for colony counts greater than 15 per spleen, the relationship between the number of cells injected and the number of colonies observed in the spleen ceases to be strictly linear, as illustrated by the data of Fig. 1.

${ }^{1}$ This research was supported in part by the Defence Research Board of Canada, under Grant DRB 9350-14 ( $\mathrm{G}$ and $\mathrm{C}$ ), and in part by the National Cancer Institute of Canada. 


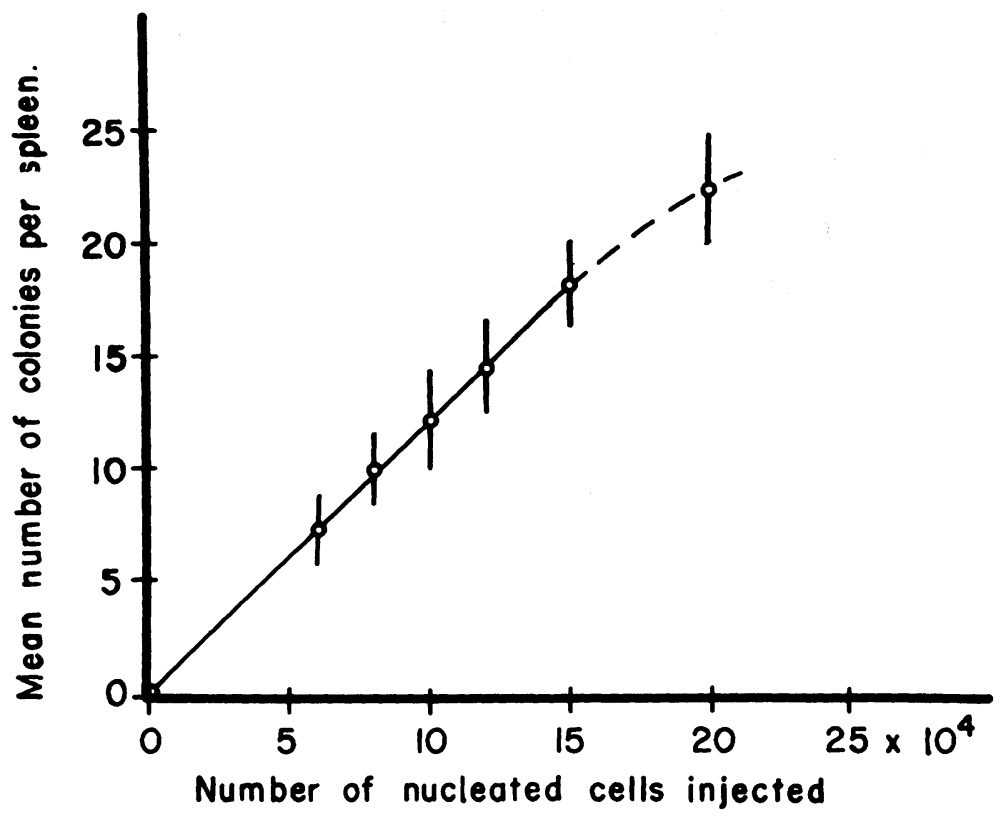

Frg. 1. The relationship between the number of nucleated cells injected and the mean number of colonies observed in the spleen after fixation in Bouin's solution. The $95 \%$ confidence limits for the points are indicated.

The curve shown in Fig. 1 is similar to that reported previously (1), but its range has been extended. The curve tends to deviate from linearity for colony numbers above 15, owing to overlap of colonies arising from crowding.

The spleens of animals receiving large numbers of colony-forming cells contain confluent colonies, which cease to be distinguishable as nodules when the normal splenic architecture is restored.

As indicated in Fig. 1, no colonies have been observed in the spleens of the few animals that have survived for 10 days after receiving 900 to 1000 rads but no injection of marrow cells. However, when lower doses of total-body radiation are used, colonies similar to those described above are observed in the spleens of animals that have not received a marrow transplant. A study of the relationship between the number of these colonies, derived from the animal's own colony-forming cells, and radiation dose, is at present under way.

The histological features of the colonies have been described (1). They consist of hemic cells in which erythroblasts, granulocytes, and megakaryocytes can be recognized. It is probable that each colony derives from a very few cells, and possibly from a single cell (1). However, as the entity in the marrow suspension that gives rise to a colony in the spleen has not yet been identified, it is referred to as a 
colony-forming unit (CFU). The ratio of the number of CFU to the number of nucleated cells injected (the CFU ratio) provides a measure of the proliferative capacity of the cells in a marrow suspension. The CFU ratio usually lies between 10 and 15 colonies per $10^{5}$ cells for normal marrow suspensions.

\section{Donor and Recipient Mice}

In most experiments $\mathrm{C} 57 \mathrm{Bl} / \mathrm{Ha}$ or $\mathrm{C} 3 \mathrm{Hf} / \mathrm{Ha}$ mice bred in the colony of the Ontario Cancer Institute, or the $\mathrm{F} 1$ hybrid between these strains, were used. As previously reported, colony-forming cells from these strains are similar in their response to radiation (1). In some experiments, pathogen-free Swiss mice (obtained from Darrow Laboratories, New York) served both as recipients and donors. The results obtained in these experiments were similar to those obtained with inbred mice.

\section{The Radiation Survival Curve for Colony-Forming Cells}

The dependence of the CFU ratio on radiation dose may be used to construct a radiation survival curve (1). Because the CFU ratio decreases as the dose of radiation is increased, it is necessary to increase the number of nucleated cells injected in order to obtain an adequate number of colonies in the spleen. It is impractical to use cell numbers greatly in excess of $10^{7}$ cells per recipient mouse, and therefore, the range of cell survival that can be studied by the method is limited to approximately 2.5 powers of ten.

To satisfy the requirements of some experiments, certain modifications were made in the technique outlined above. These will be described with the presentation of results.

\section{Irradiation Procedures}

Recipient mice were irradiated in groups of 30 in a rotating circular Lucite drum $30 \mathrm{~cm}$ in diameter, located $65 \mathrm{~cm}$ from the target of $280-\mathrm{kvp} \mathrm{X}$-ray generator, operated at 20-ma tube current, with filtration yielding a HVL of $1.12 \mathrm{~mm} \mathrm{Cu}$. The exposure dose rate was approximately $100 \mathrm{r} / \mathrm{min}$. For some experiments $200-\mathrm{kvp}$ $\mathrm{X}$-Rays, HVL $1 \mathrm{~mm} \mathrm{Cu}$, were used.

Marrow cell irradiations both in vivo and in vitro were carried out with $\mathrm{Co}^{60}$ $\gamma$-rays. In vivo irradiations were performed on groups of 10 mice in a circular Lucite holder $20 \mathrm{~cm}$ in diameter, placed on a revolving turntable beneath the $\mathrm{Co}^{60}$ source. For most experiments, an absorbed dose rate of 50 to $55 \mathrm{rads} / \mathrm{min}$ was used. In vitro irradiations were performed on marrow cell suspensions in 12-ml stoppered polyethylene tubes, $1.7 \mathrm{~cm}$ in diameter, placed in a Lucite holder (wall thickness $0.5 \mathrm{~cm}$ ) with their long axes perpendicular to the radiation beam. The tubes were agitated immediately before irradiation in order to keep the cells in suspension and to provide adequate aeration. For most experiments, an absorbed dose rate of $\mathbf{2 8 5}$ 
to $330 \mathrm{rads} / \mathrm{min}$ was used. In some experiments, in vivo and in vitro irradiations were carried out at the same dose rate, as will be described under Results.

Dosimetry was based on air ionization measurements made with a Philips vibrating-reed electrometer and on absorbed dose measurements utilizing the ferrous ammonium sulfate dosimeter (3). A $G$ value of 15.5 ferric ions produced per $100 \mathrm{ev}$ of energy absorbed was assumed.

\section{RESULTS}

Sensitivity of the Colony-Forming Ability of Marrow Irradiated in Vitro

A survival curve for colony-forming units (CFU) in marrow irradiated in vitro with $\gamma$-rays from $\mathrm{Co}^{60}$ has already been reported (1). The accumulated results from these and further experiments are shown in Fig. 2 (closed circles). In the figure, the 95\% confidence limits for each of the experimental points are shown. The portion of the survival curve which is linear on a semilogarithmic plot has been fitted to the experimental points by the method of least squares. The curve thus obtained yielded a $D_{0}$ (the radiation dose required to reduce the surviving fraction by a factor of 0.37 , measured on the exponential portion of the curve) of $105 \pm 13$ rads, and an extrapolation number (4) of 2.5. This $D_{0}$ is slightly less than the value of 115 rads reported previously (1). The limits quoted represent the $95 \%$ confidence limits of the $D_{0}$.

\section{The in Vivo Radiation Sensitivity of the Colony-Forming Ability of Marrow}

Irradiation within the mouse of aliquots of a single marrow suspension was accomplished by a modification of the assay technique, as follows. The recipient mice received a part of the usual dose of 900 to 1000 rads. After this, an intravenous injection of marrow cells from the suspension under test was given. Two to three hours thereafter the balance of the required radiation dose was administered to the mice by using $\mathrm{Co}^{60} \gamma$-rays. In this way the recipient animals received the usual heavy dose of radiation required to suppress endogenous colony-formers, but the administered cells received only the dose delivered in the second exposure. Thereafter the mice were kept for 10 days, the survivors killed, and their spleens examined for colonies. The design of a typical experiment, and the results obtained, are shown in Table I.

Data accumulated from several experiments of this design are shown in Fig. 3 (closed circles) and compared with the survival curve obtained for irradiation of cells in vitro. The upper curve, without points, is the in vitro survival curve shown in Fig. 2. The means and 95\% confidence limits for the points obtained for irradiation in vivo are shown. These all fall below the in vitro survival curve. The best fit to the exponential portion of the curve yielded a $D_{0}$ of $95 \pm 9$ rads and an extrapolation number of 1.5. Statistical analysis indicated that the two values for $D_{0}$ (105 and 95) obtained for cells irradiated in vitro and in vivo did not differ 


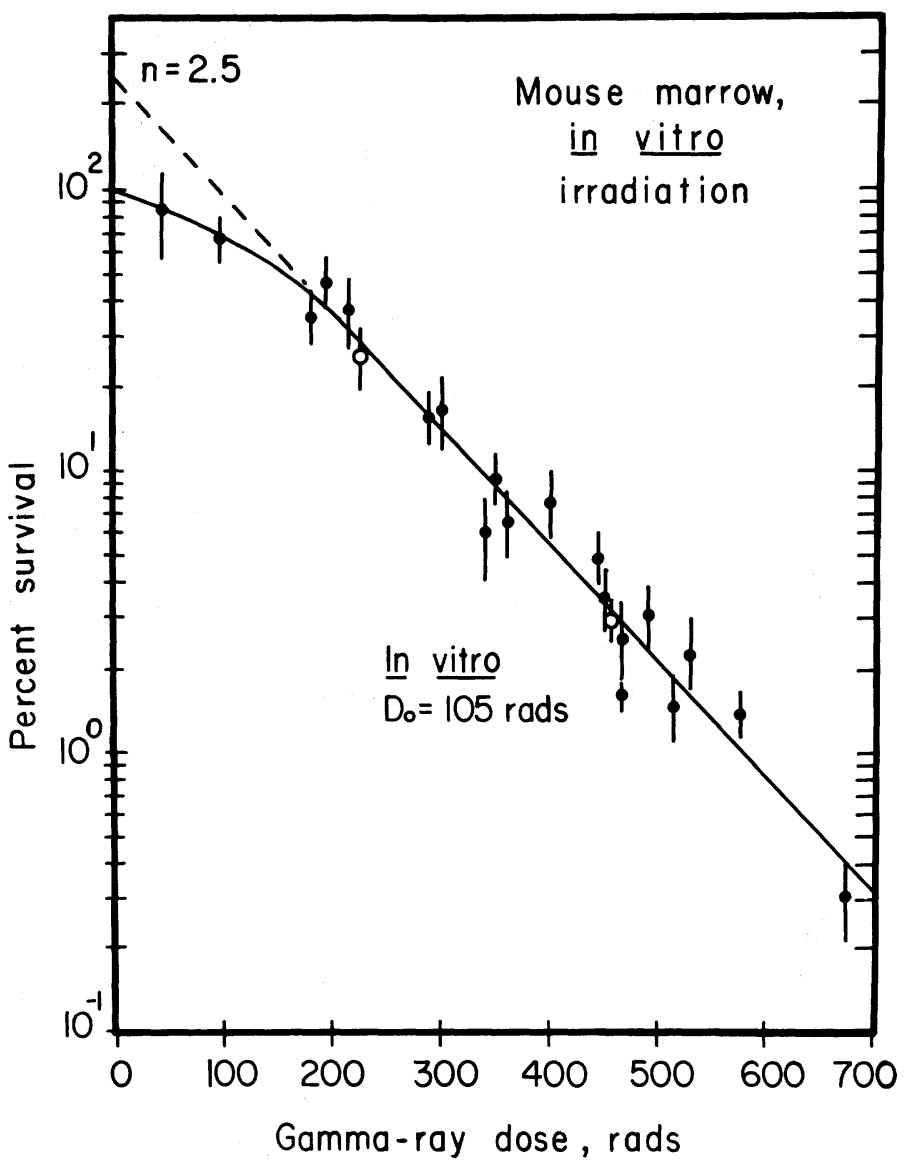

FIg. 2. Gamma-ray survival curve for the colony-forming ability of mouse bone marrow cells irradiated as a cell suspension in vitro. Closed circles: irradiated in air. Open circles: irradiated in $95 \% \mathrm{O}_{2}, 5 \% \mathrm{CO}_{2}$. The extrapolation number $(n)$ and $D_{0}$ are indicated.

significantly at the $5 \%$ level. However, the difference in the extrapolation numbers of the two curves was highly significant at the $1 \%$ level, suggesting that this difference accounts for the greater survival of cells irradiated in vitro, compared with cells irradiated in vivo.

The experimental design described above was useful because it permitted irradiation in vivo of aliquots of the same marrow suspension. However, before it could be accepted as a true measure of the radiation sensitivity of marrow cells functioning in their normal environment, it was necessary to do experiments in which marrow cells were irradiated in situ by exposing normal mice to whole-body irradiation. In these experiments, groups of 10 to 20 female donors of the same 


\section{TABLE I}

Survival of Colony-Forming Units (CFU) after

IRRADIATION in Vivo WITH $\mathrm{Co}^{60} \gamma$-RAYS

\begin{tabular}{|c|c|c|c|c|c|c|c|}
\hline Group & $\begin{array}{c}\text { First } \\
\text { irradia- } \\
\text { tion, } \\
X-\text { rays } \\
(\text { rads })\end{array}$ & $\begin{array}{c}\text { Number of } \\
\text { nucleated } \\
\text { cells } \times \\
10^{-5}\end{array}$ & $\begin{array}{c}\text { Second } \\
\text { irradia- } \\
\text { tion, } \\
\gamma-\text { rays } \\
(\text { rads })\end{array}$ & $\begin{array}{l}\text { Number } \\
\text { of } \\
\text { spleens }\end{array}$ & Mean CFU & $\begin{array}{l}C F U / 10^{5} \\
\text { cells }\end{array}$ & Survival $(\%)$ \\
\hline 1 & 950 & 1 & 0 & 22 & $18.9 \pm 3.4^{\mathrm{a}}$ & 18.9 & $100 \pm 8^{b}$ \\
\hline 2 & 825 & 3 & 125 & 19 & $23.8 \pm 5.3$ & 7.9 & $42 \pm 5.4$ \\
\hline 3 & 700 & 10 & 250 & 27 & $23.9 \pm 4.7$ & 2.4 & $13 \pm 1.4$ \\
\hline 4 & 575 & 30 & 375 & 27 & $16.9 \pm 5.2$ & 0.56 & $3.0 \pm 0.42$ \\
\hline 5 & 450 & 100 & 500 & 19 & $12.1 \pm 4.7$ & 0.12 & $0.63 \pm 0.13$ \\
\hline
\end{tabular}

a Sample standard deviation.

b $95 \%$ confidence limits.

TABLE II

Survival of CFU Irradiated in Situ in the Femora of Living Mice

\begin{tabular}{cccccccccc}
\hline Group & $\begin{array}{c}\gamma \text {-ray } \\
\text { dose } \\
\text { (rads) }\end{array}$ & $\begin{array}{c}\text { Cells per } \\
\text { two } \\
\text { femora } \\
\times 10^{-7}\end{array}$ & $\begin{array}{c}\text { Number } \\
\text { of cells } \\
\text { injected } \\
\times 10^{-5}\end{array}$ & $\begin{array}{c}\text { Number } \\
\text { of } \\
\text { spleens }\end{array}$ & Mean CFU & $\begin{array}{c}\text { CFU per } \\
10^{5} \text { cells }\end{array}$ & $\begin{array}{c}\text { Observed } \\
\text { survival (\%) }\end{array}$ & $\begin{array}{c}\text { Expected } \\
\text { survival (\%) }\end{array}$ \\
\hline 1 & 0 & 1.3 & 1 & 5 & $10.1 \pm 2.4^{\mathrm{b}}$ & 10.1 & $100 \pm 30^{\mathrm{c}}$ & 100 \\
2 & 185 & 1.5 & 5 & 7 & $11.5 \pm 2.9$ & 2.3 & $23 \pm 7.4$ & 21 \\
3 & 324 & 1.2 & 23 & 12 & $17.2 \pm 7.0$ & 0.75 & $7.4 \pm 2.5$ & 4.6 \\
4 & 438 & 1.5 & 76 & 20 & $145 \pm 5.7$ & 0.19 & $1.9 \pm 0.55$ & 1.4 \\
\hline
\end{tabular}

a Obtained from the survival curve of Fig. 3.

b Sample standard deviation.

c $95 \%$ confidence limits.

age and strain were irradiated with different doses of $\gamma$-rays. Immediately afterward the mice were killed, and their femoral marrow was pooled. The CFU ratio of the suspensions thus obtained was measured by injection of aliquots of the suspensions into heavily irradiated recipients in the usual way. The results of a typical experiment are given in Table II and, with others obtained in this way, are included in Fig. 3 as open circles.

The survivals expected from the survival curve of Fig. 3 for the radiation doses used in the experiment are included in Table II. No statistically significant difference exists between these values and those obtained with cells irradiated before extraction from the donor mice $(P=0.25)$.

These experiments support the suggestion that the in vivo survival curve obtained by the experimental design illustrated in Table $\mathrm{I}$ is valid for colony-forming cells in marrow irradiated in vivo under physiological conditions and is different 


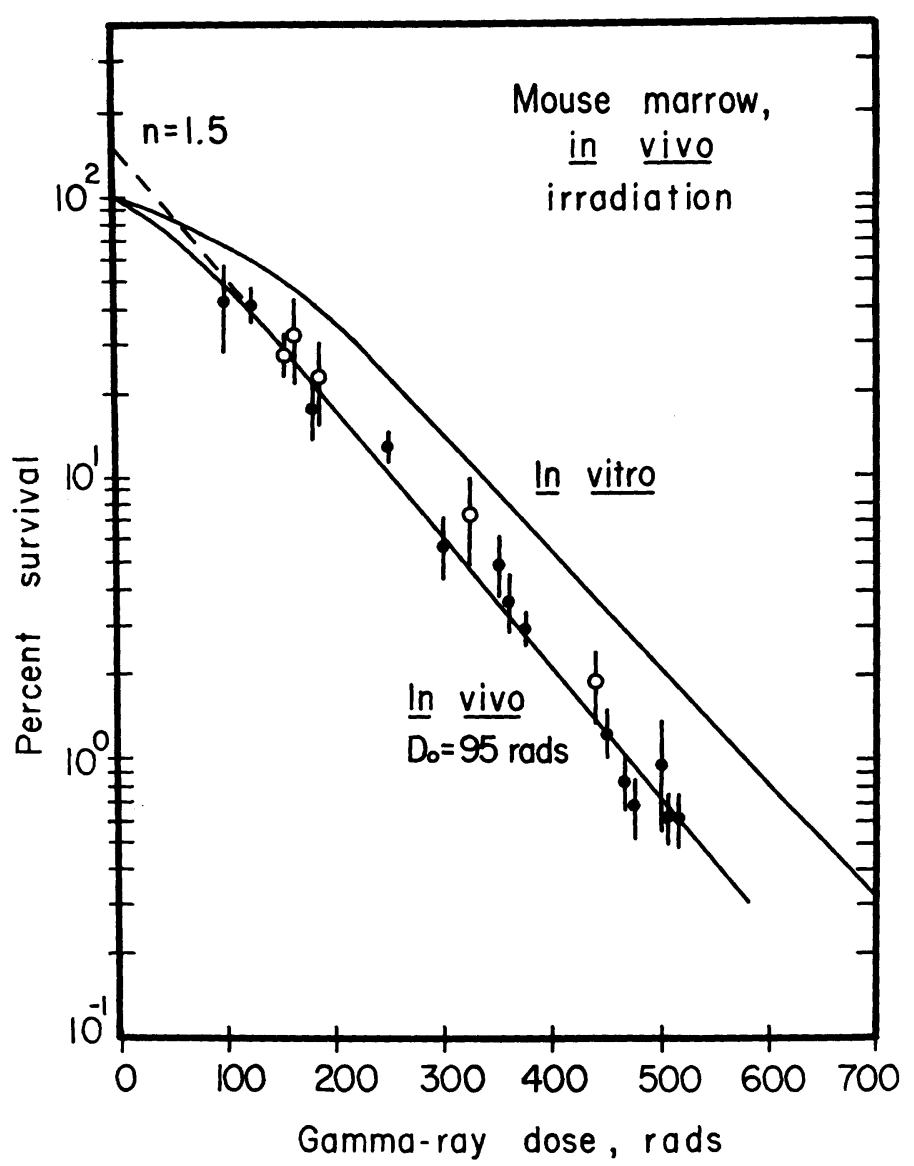

FIG. 3. Gamma-ray survival curve for the colony-forming ability of mouse marrow cells irradiated in vivo. Closed circles: irradiated after injection into the recipient mice. Open circles: irradiated in situ in the femora of living donor mice. The extrapolation number $(n)$ and $D_{0}$ are indicated.

from the survival curve obtained for the same cells irradiated in vitro. Possible explanations for this difference will now be considered.

Effect of Oxygenation in Vitro

It seemed possible that more CFU survived irradiation in suspension than irradiation within the animal because the cells in suspension were not so well oxygenated as the cells in the animal. In order to test this possibility, an experiment was done in which oxygen $\left(95 \% \mathrm{O}_{2}, 5 \% \mathrm{CO}_{2}\right)$ was bubbled vigorously through the suspension during the irradiation. No way was found by which loss of cells during the bubbling procedure could be avoided, even though an antifoaming agent (Dow Corning Antifoam AF emulsion, Dow Corning Corp., Midland, Michi- 
TABLE III

Survival of CFU Irradiated in Vitro in the Presence of $95 \% \mathrm{O}_{2}+5 \% \mathrm{CO}_{2}$

\begin{tabular}{|c|c|c|c|c|c|c|c|c|}
\hline Group & $\begin{array}{c}\gamma-r a y \\
\text { dose } \\
\text { (rads) }\end{array}$ & $\begin{array}{c}\text { Cell loss } \\
\text { on } \\
\text { bubbling } \\
(\%)\end{array}$ & $\begin{array}{c}\text { Number of } \\
\text { cells injected } \\
\times 10^{-5}\end{array}$ & $\begin{array}{c}\text { Number } \\
\text { of } \\
\text { spleens }\end{array}$ & Mean CFU & $\underset{10^{5} \mathrm{cells}}{C F U}$ & Observed survival (\%) & $\begin{array}{c}\text { Expected } \\
\text { survival } \\
(\%)^{\mathbf{a}}\end{array}$ \\
\hline 1 & 0 & 25 & 1.0 & 10 & $17.2 \pm 3.2^{\mathrm{b}}$ & 17.2 & $100 \pm 13^{c}$ & 100 \\
\hline 2 & 225 & 26 & 2.2 & 11 & $9.9 \pm 3.4$ & 4.5 & $26 \pm 6.5$ & 29 \\
\hline 3 & 450 & 28 & 49 & 22 & $26.7 \pm 3.8$ & 0.55 & $3.2 \pm 0.40$ & 3.2 \\
\hline 4 & 450 & 28 & 38 & 17 & $18.2 \pm 5.3$ & 0.48 & $2.8 \pm 0.54$ & 3.2 \\
\hline
\end{tabular}

a Obtained from the survival curve of Fig. 2 .

b Sample standard deviation.

c $95 \%$ confidence limits.

gan) added to the cell suspension (dilution factor $10^{-4}$ ) suppressed excessive foam formation. Because of this loss of cells, each aliquot of the suspension was bubbled and irradiated at maximum cell concentration, and the unirradiated control aliquot was bubbled for a similar length of time. The results of this experiment are shown in Table III, and the surviving fractions listed in the table are included in Fig 2 (open circles). Comparison of the experimental values of Table III with the percentage survivals to be expected on the basis of the survival curve of Fig. 2 indicates that no statistically significant difference exists $(P>0.5)$. This experiment thus suggests that partial anoxia cannot account for the greater survival of cells irradiated in vitro compared with those irradiated in vivo.

This result does not mean that the radiosensitivity of CFU is independent of oxygen concentration. Preliminary experiments have demonstrated that cells irradiated under anoxic conditions do show the two- to threefold decrease in radiosensitivity found in other systems (5).

\section{Effect of Dose Rate on Survival of Cells Irradiated in Vitro}

In most experiments, cells were irradiated in vitro at a higher dose rate than cells irradiated in vivo. In order to test the possibility that the observed difference between the two survival curves was due to the difference in dose rate, aliquots of the same suspension were irradiated in vivo and in vitro at the same distance from the source, i.e., at the same dose rate, and under conditions as nearly identical as possible. The results of the experiment are shown in Table IV. The results are in good agreement with the data of Figs. 2 and 3. Approximately twice as many cells survived the same dose of $\gamma$-rays in vitro as survived irradiation in vivo under nearly identical radiation conditions.

\section{The Transition from in Vitro to in Vivo Sensitivity}

The preceding experiment indicated that the observed difference between the in vitro and in vivo survival curves did not arise from differences in dose rate. 
TABLE IV

Survival of CFU Irradiated Under Similar Geometry

(Dose rate $55 \mathrm{rads} / \mathrm{min}$ )

\begin{tabular}{|c|c|c|c|}
\hline \multicolumn{2}{|c|}{ Irradiation in vitro } & \multicolumn{2}{|c|}{ Irradiation in vivo } \\
\hline $\begin{array}{c}\gamma-\text { ray dose } \\
(\text { rads })\end{array}$ & Survivala $^{\mathrm{a}}(\%)$ & $\begin{array}{c}\gamma-r a y \\
\text { dose (rads) }\end{array}$ & Survivala $^{\text {a }}(\%)$ \\
\hline 0 & $100 \pm 16$ & 0 & $100 \pm 16$ \\
\hline 179 & $36 \pm 8$ & 179 & $18 \pm 3.9$ \\
\hline 359 & $6.7 \pm 1.8$ & 359 & $3.7 \pm 0.84$ \\
\hline 513 & $1.5 \pm 0.4$ & 513 & $0.62 \pm 0.14$ \\
\hline
\end{tabular}

a $95 \%$ confidence limits are indicated.

However, it was possible that uncertainties in dosimetry, due to differences in the absorption of radiation in the mouse as compared to the cell suspension, might be the basis for the observed difference in cell survival. Should this be the case, the survival of cells at a given dose of radiation should change abruptly when the cells are introduced into the mouse and the new conditions for absorption of energy are established. On the other hand, if the change in sensitivity is the result of changed metabolic or environmental conditions acting on the cell, it might be expected that the transition from the in vitro to the in vivo sensitivity might occupy a period of time. For this reason experiments were performed in which marrow cells were irradiated in vivo at different intervals after injection into irradiated hosts. The results of two experiments are given in Table $\mathrm{V}$.

These results indicate that the sensitivity of the cells does not change abruptly immediately after injection into the mice; a period of time is required for the transition between the in vitro sensitivity and the in vivo sensitivity. Though the

TABLE V

The Transition from in Vitro to in Vivo Radiation Sensitivity

(Radiation dose, 465 rads)

\begin{tabular}{ccc}
\hline Irradiation & $\begin{array}{c}\text { Experiment 1, } \\
\text { survival (\%) }\end{array}$ & $\begin{array}{l}\text { Experiment 2, } \\
\text { survival (\%) }\end{array}$ \\
\hline $\begin{array}{c}\text { In vitro } \\
\text { Interval after } \\
\text { injection of } \\
\text { cells (min) }\end{array}$ & $1.6 \pm 0.23$ & $2.6 \pm 0.77$ \\
& & \\
2 & $2.4 \pm 0.63$ & $1.4 \pm 0.38$ \\
10 & $2.1 \pm 0.59$ & $1.4 \pm 0.49$ \\
20 & $1.2 \pm 0.30$ & $1.1 \pm 0.29$ \\
300 & $0.84 \pm 0.18$ & $0.68 \pm 0.16$ \\
\hline
\end{tabular}

a $95 \%$ confidence limits are indicated. 
precision of the data is not sufficient to allow any more than a crude estimate to be made of the rate of change of the surviving fraction from the in vitro to the in vivo sensitivity, the results are compatible with the assumption that half the total change is accomplished in about 10 minutes.

Since the sensitivity of cells does not change abruptly on injection into the mouse, it appears that the difference in survival of cells irradiated in vivo compared to cells irradiated in vitro is probably not due to physical factors.

\section{DISCUSSION}

In previous experiments $(6)$, using a less sensitive measure of the proliferative capacity of bone marrow, no difference between the effects of radiation in vivo and in vitro was found. Similarly, Hewitt and Wilson (7), using a transplantable murine leukemia, found that cells irradiated in vitro and assayed in vivo had a similar sensitivity to cells irradiated and assayed in vivo. On the other hand, Philpott, Shaeffer, and Tolmach, ${ }^{2}$ using chick embryo cells, found that cells irradiated in ovo were slightly more resistant than cells irradiated in vitro. The experiments reported in this paper indicate that a small but statistically significant difference in survival does exist between marrow irradiated in vitro and marrow irradiated in vivo. This finding does not affect the observation that the $D_{0}$ for colony-forming cells from mouse bone marrow is very nearly $100 \mathrm{rads}$, since most of the difference between the in vivo and in vitro results is due to a difference in the extrapolation number.

The difference in survival for the cells irradiated in vitro and in vivo is not due to errors in absorbed dose measurements. This is demonstrated by the fact that similar survival values were obtained when cells were irradiated in vitro and when cells were irradiated within recipient mice a very short time after their injection. Thus, the differences in survival are probably due to biological effects resulting from the reaction of the cell with its environment.

A number of changes in the sensitivity of cells to ionizing radiation have been shown to be the result of changes in oxygen concentration (5). However, the results presented above suggest that this is not the explanation for the differences observed.

Two possible explanations for the difference suggest themselves. First, the effective radiation sensitivity of the cells might be less in vitro than in vivo, owing to mechanisms operating at the cellular level; for example, metabolically active cells in the animal might be more readily inactivated than resting cells outside the body. This sort of dependence of cell survival on the growth conditions after irradiation has been observed in the bacterium Escherichia coli B by Alper and Gillies (8). Alternatively, recovery processes involving changes in extrapolation number, of the type described by Elkind and Sutton (9) might be less effective in vivo than in vitro. This latter possibility is suggested by the observation that the extrapola-

${ }^{2}$ G. W. Philpott, C. W. Shaeffer, Jr., and L. J. Tolmach, personal communication (Washington University, School of Medicine, Saint Louis, Missouri). 
tion number of the in vivo survival curve is less than the extrapolation number of the in vitro curve.

Second, the decreased survival of cells irradiated in the animal might result from host influences acting on the cells after irradiation in vivo, leading to a reduction in the number with the capacity to form colonies. For instance, nonlethal radiation effects might increase the likelihood that a cell would become more differentiated, and by differentiation lose its capacity to form a colony.

The data presented above do not permit any conclusions to be drawn concerning the basis for the difference between the survival of cells irradiated in vitro and in vivo. This difference, though small in magnitude, may provide an important clue to the mechanism of action of radiation on mammalian cells.

\section{SUMMARY}

1. A survival curve for the colony-forming ability of mouse bone marrow cells irradiated in vivo with $\mathrm{Co}^{60} \gamma$-rays has been obtained. The curve may be characterized by a $D_{0}$ of 95 rads and an extrapolation number of 1.5 .

2. Comparison with the survival curve obtained for cells irradiated in vitro indicated a statistically significant difference in extrapolation number. Possible explanations for this finding are considered.

\section{ACKNOWLEDGMENTS}

We wish to thank Miss Rosemary Wyncoll, Mr. James Hicks, and Mr. Paul Csordas for their excellent technical assistance.

RECEIVED: October 12, 1961

\section{REFERENCES}

1. J. E. Till and E. A. McCulloch, A direct measurement of the radiation sensitivity of normal mouse bone marrow cells. Radiation Research 14, 213-222 (1961).

2. R. D. LILlIE, Histopathologic Technic and Practical Histochemistry, p. 46, Blakiston and Co., New York, 1954.

3. Report of the International Commission on Radiological Units and Measurements (ICRU) 1959. Natl. Bur. Standards (U. S.) Handbook No. 78 (1961).

4. T. Alper, N. E. Gillies, and M. M. Elkind, The sigmoid survival curve in radiobiology. Nature 186, 1062-1063 (1960).

5. L. H. GraY, Cellular radiobiology. Radiation Research Suppl. 1, 73-101 (1959).

6. E. A. McCulloch and J. E. TILL, The radiation sensitivity of normal mouse bone marrow cells, determined by quantitative marrow transplantation into irradiated mice. Radiation Research 13, 115-125 (1960).

7. H. B. Hewitt and C. W. Wilson, Survival curves for tumor cells irradiated in vivo. Ann. N. Y. Acad.Sci. 95, 818-827 (1961).

8. T. AlPER and N. E. Gillies, The relationship between growth and survival after irradiation of Escherichia coli strain B and two resistant mutants. J. Gen. Microbiol. 22, 113-128 (1960).

9. M. M. Elkind and H. Sutton, Radiation response of mamalian cells grown in culture. 1. Repair of X-ray damage in surviving Chinese hamster cells. Radiation Research 13, 556593 (1960). 